\title{
Síndrome metabólica em pessoas vivendo com HIV: prevalência e concordância de critérios
}

Metabolic syndrome in people living with HIV: criteria prevalence and agreement Síndrome metabólico en personas que viven con el VIH: prevalencia y concordancia de criterios

Christefany Régia Braz Costa ${ }^{1}$ id https://orcid.org/0000-0001-6124-8243

Elizabete Santos Melo ${ }^{1}$ iD https://orcid.org/0000-0002-7322-9370

Layze Braz de Oliveira ${ }^{1}$ iD https://orcid.org/0000-0001-7472-5213

Rita Simone Lopes Moreira² id https://orcid.org/0000-0003-3743-9044

Elucir Gir ${ }^{1}$ id https://orcid.org/0000-0002-3757-4900

Renata Karina Reis ${ }^{1}$ id https://orcid.org/0000-0002-0681-4721

Como citar:

Costa CR, Melo ES, Oliveira LB, Moreira RS, Gir

E, Reis RK. Síndrome metabólica em pessoas vivendo com HIV: prevalência e concordância de critérios. Acta Paul Enferm. 2021;34:AAPE00625.

DOI

http://dx.doi.org/10.37689/actaape/2021A000625

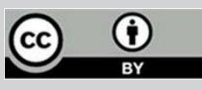

Descritores

Síndrome metabólica; HIV; Síndrome de imunodeficiência adquirida; Prevalência

Keywords

Metabolic syndrome; HIV; Acquired Immunodeficiency Syndrome; Prevalence

Descriptores

Síndrome metabólico; HIV: Síndrome de imunodeficiência adquirida; Prevalencia

\section{Submetido \\ 27 de Março de 2020 \\ Aceito \\ 2 de Dezembro de 2020}

Autor correspondente

Christefany Régia Braz Costa E-mail: christefany.costa@usp.br

\section{Resumo}

Objetivo: Identificar a prevalência da síndrome metabólica e a concordância entre os critérios do National Cholesterol Education Program Adult Treatment Panel III (NCEP-ATPIII) e da International Diabetes Federation (IDF) em pessoas vivendo com HIV.

Métodos: Estudo analítico transversal, realizado em cinco serviços especializados em município do interior paulista, de 2014 a 2016, com 340 pessoas vivendo com HIV. Variáveis sociodemográficas e clínicas necessárias para classificação da síndrome metabólica pelos critérios do NCEP-ATPIII e da IDF foram coletadas por meio de entrevistas. Para avaliar a concordância entre os critérios da SM, NCEP-ATPIII e IDF, foi utilizada a estatística first-order agreement coefficient. Para verificar a relação entre a síndrome metabólica e as variáveis do estudo, utilizou-se a regressão de Poisson com variância robusta.

Resultados: A prevalência da síndrome metabólica foi de $28,5 \%$ pelo critério NCEP-ATPIII e 39,3\% IDF. As maiores prevalências foram associadas ao sexo feminino e faixas etárias a partir dos 50 anos, enquanto que, no tempo de diagnóstico entre 2 a 10 anos, prevalências menores. A concordância entre os dois critérios foi considerada substancial.

Conclusão: A concordância substancial entre os critérios IDF e NCEP-ATPIII sugere a possibilidade de intercambio entre eles. Ademais, os resultados sinalizam para a necessidade de atenção especial dos serviços para a avaliação do perfil metabólico e identificação das pessoas vivendo com HIV que possuem alto risco cardiovascular.

\section{Abstract}

Objective: To identify the prevalence of metabolic syndrome and the agreement between the criteria of the National Cholesterol Education Program Adult Treatment Panel III (NCEP-ATPIII) and the International Diabetes Federation (IDF) in people living with HIV.

Methods: This is a cross-sectional analytical study, carried out in five specialized services in a city in the interior of São Paulo, from 2014 to 2016, with 340 people living with HIV. Sociodemographic and clinical variables necessary for classification of the metabolic syndrome by the NCEP-ATPIII and IDF criteria were collected through interviews. To assess the agreement between MS, NCEP-ATPIII and IDF criteria, the firstorder agreement coefficient statistic was used. To verify the relationship between the metabolic syndrome and the study variables, Poisson regression with robust variance was used.

Results: The prevalence of metabolic syndrome was $28.5 \%$ by the NCEP-ATPIII criterion and $39.3 \%$ IDF. The highest prevalence was associated with females and age groups from 50 years old, while, in the time of diagnosis between 2 and 10 years, lower prevalence. The agreement between the two criteria was considered substantial. 
Conclusion: The substantial agreement between the IDF and NCEP-ATPIII criteria suggests the possibility of interchange between them. Moreover, the results signal the need for special attention from services for the assessment of the metabolic profile and identification of people living with HIV who are at high cardiovascular risk.

\section{Resumen}

Objetivo: Identificar la prevalencia del síndrome metabólico y la concordancia entre los criterios del National Cholesterol Education Program Adult Treatment Panel III (NCEP-ATPIII) y de la International Diabetes Federation (IDF) en personas que viven con el VIH.

Métodos: Estudio analítico transversal, realizado en cinco servicios especializados en un municipio del interior del estado de São Paulo, de 2014 a 2016, con 340 personas que viven con el VIH. Por medio de entrevistas se recopilaron las variables sociodemográficas y clínicas necesarias para la clasificación del síndrome metabólico mediante los criterios del NCEP-ATPIII y de la IDF. Para evaluar la concordancia entre los criterios del SM, NCEP-ATPIII e IDF, se utilizó la estadística first-order agreement coefficient. Para verificar la relación entre el síndrome metabólico y las variables del estudio, se utilizó la regresión de Poisson con varianza robusta.

Resultados: La prevalencia del síndrome metabólico fue del 28,5 \% mediante el criterio NCEP-ATPIII y 39,3 \% por la IDF. Las mayores prevalencias se asociaron al sexo femenino y los grupos de edad a partir de los 50 años, mientras que hubo prevalencias menores en el tiempo de diagnóstico entre 2 y 10 años. La concordancia entre los dos criterios fue considerada sustancial.

Conclusión: La concordancia sustancial entre los criterios IDF y NCEP-ATPIII sugiere la posibilidad de intercambio entre ellos. Además, los resultados señalan la necesidad de una atención especial de los servicios para evaluar el perfil metabólico e identificar a las personas que viven con el VIH con alto riesgo cardiovascular.

\section{Introdução}

Os avanços na terapia antirretroviral combinada (TARV) e seu acesso universal proporcionou grande impacto para as pessoas vivendo com o HIV (PVHIV). Os pacientes que possuíam perspectivas limitadas passaram a viver uma nova fase do tratamento. A adesão a medicação tem sido o principal contribuinte para o declínio das mortes relacionadas à aids, aumento da sobrevida, melhora na qualidade de vida, diminuição da transmissão e supressão da atividade viral, possibilitando assim viver com o HIV numa perspectiva de condição crônica. ${ }^{(1,2)}$

No mundo, até 2018, foi estimado que cerca de 37,9 milhóes PVHIV e, entre os diagnosticados, cerca de $62 \%$ estão em tratamento com antirretrovirais (ARV). Já no Brasil, há cerca de 900.000 PVHIV e de acordo com o Ministério da Saúde $66 \%$ destes tem acesso a medicação. ${ }^{(3-5)}$

A despeito das possibilidades alvissareiras proporcionadas pelo uso da TARV, os pacientes podem experimentar alteraçóes metabólicas e toxicidades específicas atreladas ao uso dessa medicação. Essas alteraçōes metabólicas, com frequência, se enquadram nos critérios para identificar a presença da síndrome metabólica (SM). (6) A SM apresenta-se mundialmente em ascensão e tem sido considerada uma entidade complexa pelo fato de agregar fatores de risco cardiovascular bem estabelecidos, aumentando a mortalidade geral. ${ }^{(5)}$ Há evidências de que a SM é mais prevalente entre pessoas que vivem com o HIV do que na população geral, o que requer assistência adequada da equipe de saúde. ${ }^{(1,6,7)}$

Desde a década de 80 , quando a SM foi reconhecida, incialmente denominada Síndrome $\mathrm{X}$, diversos critérios foram utilizados para defini-la. Atualmente, os critérios mais utilizados nacional e internacionalmente entre PVHIV, são o do National Cholesterol Education Program Adult Treatment Panel III (NCEP-ATPIII) e da International Diabetes Federation (IDF). Essas organizações, apesar de aplicarem os critérios de maneira diferente, concordam que os componentes básicos da SM são obesidade, resistência à insulina, dislipidemia e hipertensão. ${ }^{(8,9)}$ Em razão de haver diferentes critérios para o diagnóstico de $\mathrm{SM}$, existem também estimativas variadas na sua prevalência. Em uma meta-análise que analisou estudos sobre a prevalência da SM em PVHIV, os resultados variaram entre 17 a $31 \% .^{(9)}$

Apesar do estabelecimento da melhora da imunidade, o uso prolongado dessa terapêutica medicamentosa pode elevar o risco de desenvolvimento da SM em PVHIV, identificar essa problemática proporciona subsídio para o gerenciamento do risco cardiovascular nessa população. Diante do exposto, o presente estudo tem como objetivo identificar a prevalência da SM e a concordância entre os critérios do NCEP-ATPIII e da IDF em PVHIV. 


\section{Métodos}

Trata-se de um estudo analítico transversal, desenvolvido nos cinco ambulatórios de atendimento especializado às pessoas que vivem com HIV no município do interior paulista, no período de outubro de 2014 a outubro de 2016.

Participaram do estudo PVHIV usuárias dos serviços que atenderam aos critérios de inclusão: possuir idade igual ou superior a 18 anos; estar em uso da TARV há pelo menos seis meses; e estar em acompanhamento clínico-ambulatorial nos serviços escolhidos. Foram critérios de exclusão: indivíduos em situações de confinamento (institucionalizados e residentes em casas de apoio), gestantes e história prévia de doença cardiovascular.

Para obter o número de participantes, foi realizado cálculo amostral baseado no número de indivíduos em uso de TARV em cada serviço de referência do município estudado no ano de 2014, que somavam 1920 pacientes. Para o cálculo do tamanho amostral foi utilizado a seguinte fórmula:

$$
n=\frac{Z \alpha^{2} \cdot(P \cdot Q)}{d^{2}},
$$

onde $\mathrm{n}$ é o tamanho amostral, $\mathrm{Z}$ é a variável reduzida para um $\alpha=5 \%, P=50 \%$ e um nível de precisão $d=5 \%$, foi feita correção para uma populaçáo finita, o que resultou em 43 no ambulatório do distrito Norte; 119 do Central; 50 do Leste; 78 do Oeste e 50 do distrito Sul, totalizando um tamanho amostral de 340. A amostragem foi não-probalística, por conveniência. Assim, os participantes foram convidados a participar à medida que apareciam no serviço para atendimento.

Os dados foram coletados por meio de entrevistas individuais, com duração média de 30 minutos, antes ou após as consultas médicas e/ou de enfermagem, em salas privativas do próprio ambulatório. A coleta de dados foi realizada por equipe treinada e certificada. Utilizou-se um questionário semiestruturado elaborado para estudo. $\mathrm{O}$ questionário foi submetido à validaçáo de face e conteúdo, por quatro pesquisadores, os quais o avaliaram quanto à aceitação do questionamento, fácil compreensão, relevância dos itens, clareza da redação, presença de ambiguidades, além de sugestôes de alteraçôes. Houve apenas apreciação qualitativa em relação ao mérito das opinióes dos pesquisadores, sem publicação prévia a este estudo.

As variáveis sociodemográficas e antecedentes clínicos pessoais foram questionadas ao paciente. A circunferência abdominal (CA) e a pressão arterial (PA) foram mensuradas após a entrevista, e os dados referentes ao tempo de TARV, esquema terapêutico e exames laboratoriais, coletados no prontuário. Dos exames, foram registrados os que tinham a data mais próxima de ingresso do paciente no estudo e descartados exames realizados no prazo maior que um ano.

A pressão arterial sistólica (PAS) e diastólica (PAD) foram avaliadas com técnica auscultatória, registrada no braço, com esfigmomanômetro aneroide, manômetro calibrado e braçadeira de tamanho apropriado. A avaliação ocorreu após cinco minutos em repouso, na posiçáo sentada, o tronco apoiado no encosto da cadeira, pernas descruzadas e apoiadas ao chão. ${ }^{(10)}$

A CA foi medida com fita métrica inextensível, em posiçâo ortostática, com a planta do pé apoiada no solo, orientada para que ficasse com o abdome relaxado, afastando as roupas da região da aferição para melhor precisão da mensuração. A medida foi aferida no ponto médio entre a última costela e a crista ilíaca, no final do movimento respiratório da expiração. ${ }^{(11)}$

As medições bioquímicas realizadas nos SAE referente à glicose venosa em jejum (GVJ), High Density Lipoproteins Cholesterol (HDL-c) e triglicérides (TG), foram realizadas por método enzimático em equipamento automatizado $\left(\mathrm{Rxl} \mathrm{Max}^{\oplus}\right)$, em laboratório central vinculado a secretaria municipal de saúde, com amostras de soro venoso, sob orientação de jejum durante por 12 horas, conforme rotina.

Para a classificação da SM utilizou-se o critério do NCEP-ATP III e da IDF. No NCEP-ATP III revisado os participantes precisam ter pelo menos três dos cinco componentes: PA $(\geq 130 / 85 \mathrm{~mm} / \mathrm{Hg}$ ou uso de anti-hipertensivo); CA ( $\geq 102 \mathrm{~cm}$ para homens e $\geq 88 \mathrm{~cm}$ para mulheres); TG $(\geq 150 \mathrm{mg} / \mathrm{dl}$ ou uso de hipolipemiantes), GVJ ( $\geq 100 \mathrm{mg} / \mathrm{dl}$ ou 
uso de hipoglicemiante) e/ou HDL-c $(<40 \mathrm{mg} / \mathrm{dl}$ para homens e $<50 \mathrm{mg} / \mathrm{dl}$ para mulheres ou uso de hipolipemiantes). ${ }^{(12)}$

O critério da IDF assume os mesmo valores para a PA, TG, GVJ e HDL-c, difere apenas na circunferência abdominal (CA) (mulheres $\geq 80 \mathrm{~cm}$ e homens $\geq 90$ $\mathrm{cm}) .{ }^{(12)}$ Pelo critério da IDF a presença de alteração na CA é obrigatória, somado a dois outros critérios, e deve-se respeitar parâmetros étnicos. Por não haver estudos importantes que estabeleçam parâmetros na América central e do Sul, recomenda-se a utilização da referência dos sul-asiáticos, já descrita. ${ }^{(13,14)}$

Assim, as variáveis estudadas foram as sociodemográficas: sexo (masculino, feminino), faixa etária em anos (20-29, 30-39, 40-49, 50-59, $\geq 60$ ), escolaridade em anos $(\leq 8,>8)$; e as variáveis clínicas: tempo de diagnóstico em anos $(\leq 1,2-4,5-10,>10)$, tempo de uso da TARV em anos $(\leq 1,2-4,5-10,>10)$, carga viral em cópias/ml $(>40, \leq 40)$, PAS e PAD $(\mathrm{mmHg})$, CA $(\mathrm{cm})$, TG $(\mathrm{mg} / \mathrm{dl})$, GVJ (mg/dl), HDL-c (mg/ dl), Hipertensão Arterial Sistêmica (HAS) (sim, não), Diabetes Melittus (DM) (sim, não), SM (sim, não), uso de anti-hipertensivo (sim, não), uso de hipolipemiante (sim, não), uso de hipoglicemiante (sim, não).

Para análise dos dados utilizou-se estatística descritiva (média e desvio padrão) para variáveis contínuas. O teste T de Student para amostras independentes foi utilizado para comparar componentes da SM (variáveis contínuas) entre homens e mulheres. Para estimar a Razão de Prevalência ajustada utilizou-se a regressão de Poisson com variância robusta. $\mathrm{Na}$ avaliação da medida de significância estatística, utilizou-se o teste de Wald. Nessas análises utilizou-se o software Statistical Package for Social Science (SPSS), versão 22.0. Para avaliar a concordância entre os critérios da SM, NCEP-ATPIII e IDF, foi utilizada a estatística AC1 (first-order agreement coefficient). A estatística AC1 tem como vantagens sobre o índice de concordância Kappa a resistência com relação à homogeneidade marginal e o traço de prevalência, além de possuir a mesma interpretação da estatística Kappa [Pobre/sem concordância $(<0,0)$; leve $(0,0-0,2)$; razoável $(0,21-0,40)$; moderada $(0,41-0,60)$; substancial $(0,61-0,80)$; quase perfeita $(0,81-1,00)] .{ }^{(15)}$ Utilizou-se para as últimas análises citadas, o programa de software $R$ versão
3.5.1. Em todas as análises realizadas no estudo, adotou-se um intervalo de confiança de $95 \%$ e nível de significância de 0,05 . O estudo foi aprovado pelo Comitê de Ética em Pesquisa. As PVHIV que aceitaram participar do estudo assinaram o Termo de Consentimento Livre e Esclarecido.

\section{Resultados}

Participaram do estudo 340 PVHIV em uso de TARV acompanhadas ambulatoriamente. Em relação à caracterização dos entrevistados, verificou-se que 197 (57,9\%) pertenciam ao sexo masculino, com média de idade de 44,35 $\pm 11,7$, variando de 20 a 75 anos. A maior concentração foi na faixa etária de 40-49 anos de idade, com 34,1\% e tinham mais de oito anos de estudo (50,9\%).

Quanto às variáveis clínicas, identificou-se que 53,8\% foram diagnosticados com HIV a menos de dez anos. Esse mesmo período -menos de dez anosfoi predominante quando avaliado o tempo de tratamento antirretroviral, 65\% dos entrevistados. A da carga viral era predominantemente indetectável $(<40$ cópias $/ \mathrm{ml}), 80,9 \%$. A partir dos critérios de SM utilizados obteve-se prevalência de 28,5\% (NCEP-ATPIII) e de 39,3\% (IDF).

As variáveis que mantiveram associação após a regressão de Poisson com maiores prevalências para a SM (Tabela 1) foram, pelo critério da IDF, a faixa etária de 50-59 (RP=2,46; IC:1,17-5,21) e 60 ou mais $(\mathrm{RP}=3,04$; IC:1,43-6,47); e o tempo de diagnóstico entre 2-4 anos ( $\mathrm{RP}=0,33$; IC:0,12-0,88) e 5-10 anos ( $\mathrm{RP}=0,40$; IC:0,14-1,10) representaram menores prevalências para a síndrome. No critério do NCEP-ATPIII o mais prevalente foi o sexo feminino $(\mathrm{RP}=1,89$; IC: 1,32-2,70) (Tabela 1).

Em relação à média dos componentes da SM avaliados por sexo (Tabela 2 ), nas mulheres os valores de glicemia venosa de jejum $(\mathrm{p}=0,02)$ e HDL-c $(\mathrm{p}=0,001)$ foram maiores, enquanto a PAS foi maior nos homens $(\mathrm{p}=0,01)$.

Conforme a distribuição da prevalência dos componentes da SM , segundo o critério do NCEPATPIII os componentes metabólicos alterados que atingiram maiores percentuais foram a HDL-c 
Tabela 1. Prevalência, razão de prevalência e intervalos de confiança entre a síndrome metabólica em e as variáveis do estudo, em pessoas vivendo com HIV $(n=340)$

\begin{tabular}{|c|c|c|c|c|c|c|}
\hline \multirow[b]{2}{*}{ Variáveis } & \multicolumn{3}{|c|}{ Critério NCEP-ATPIII } & \multicolumn{3}{|c|}{ Critério IDF } \\
\hline & $\begin{array}{l}\text { Prevalência } \\
\text { n(\%) }\end{array}$ & $\begin{array}{c}\mathrm{RP} \\
\text { (IC95\%) }\end{array}$ & $p$-value ${ }^{*}$ & $\begin{array}{c}\text { Prevalência } \\
\mathrm{n}(\%)\end{array}$ & $\begin{array}{c}\mathrm{RP} \\
(\mathrm{IC} 95 \%)\end{array}$ & $p$-value ${ }^{*}$ \\
\hline \multicolumn{7}{|l|}{ Sexo } \\
\hline Masculino & $35(36,1)$ & 1,0 & & $64(47,8)$ & 1,0 & \\
\hline Feminino & $62(63,9)$ & $1,89(1,32-2,70)$ & $<0,001$ & $70(52,2)$ & $1,29(0,99-1,69)$ & 0,057 \\
\hline 20-29 & $3(3,1)$ & 1,0 & & $7(5,2)$ & 1,0 & \\
\hline 30-39 & $14(14,4)$ & $1,90(0,60-5,99)$ & 0,269 & $21(15,7)$ & $1,88(0,89-3,97)$ & 0,095 \\
\hline $40-49$ & $28(28,9)$ & $1,50(0,46-4,86)$ & 0,499 & $43(32,1)$ & $1,81(0,85-3,85)$ & 0,119 \\
\hline $50-59$ & $36(37,1)$ & $2,9(0,91-9,27)$ & 0,070 & $42(31,3)$ & $2,46(1,17-5,21)$ & 0,018 \\
\hline 60 ou mais & $16(16,5)$ & $9,8(0,88-9,13)$ & 0,810 & $21(15,7)$ & $3,04(1,43-6,47)$ & 0,004 \\
\hline \multicolumn{7}{|c|}{ Escolaridade (em anos) } \\
\hline$\leq 8$ & $60(61,9)$ & 1,0 & & $79(59,0)$ & 1,0 & \\
\hline$>8$ & $37(38,1)$ & $1,09(0,78-1,53)$ & 0,581 & $55(41,0)$ & $1,16(0,88-1,52)$ & 0,278 \\
\hline \multicolumn{7}{|c|}{ Tempo de diagnóstico (em anos) } \\
\hline$\leq 1$ & $5(5,1)$ & 1,0 & & $17(12,7)$ & 1,0 & \\
\hline $2-4$ & $9(9,3)$ & $0,57(0,12-2,65)$ & 0,482 & $14(10,4)$ & $0,33(0,12-0,88)$ & 0,027 \\
\hline $5-10$ & $18(18,6)$ & $0,62(0,15-2,46)$ & 0,497 & $25(18,7)$ & $0,33(0,13-0,88)$ & 0,027 \\
\hline$>10$ & $65(67,0)$ & $1,05(0,26-4,22)$ & 0,936 & $78(58,2)$ & $0,40(0,14-1,10)$ & 0,780 \\
\hline \multicolumn{7}{|l|}{ Tempo de TARV } \\
\hline$\leq 1$ & $8(8,3)$ & 1,0 & & $22(16,4)$ & 1,0 & \\
\hline $2-4$ & $14(14,4)$ & $1,85(0,58-5,92)$ & 0,296 & $19(14,2)$ & $1,74(0,72-4,18)$ & 0,214 \\
\hline $5-10$ & $27(27,8)$ & $2,34(0,81-6,77)$ & 0,116 & $32(23,9)$ & $2,00(0,83-4,83)$ & 0,120 \\
\hline$>10$ & $48(49,5)$ & $1,84(0,63-5,36)$ & 0,262 & $61(45,5)$ & $2,14(0,85-5,41)$ & 0,106 \\
\hline \multicolumn{7}{|c|}{ Carga viral (cópias/ml) } \\
\hline$\leq 40$ & $85(87,6)$ & 1,0 & & $112(83,6)$ & 1,0 & \\
\hline$>40$ & $12(12,4)$ & $1,59(0,93-2,72)$ & 0,088 & $22(16,4)$ & $1,17(0,80-1,71)$ & 0,405 \\
\hline
\end{tabular}

NCEP-ATPIII - National Cholesterol Education Program Adult Treatment Panel IIl; IDF - International Diabetes Federation; RP - Razão de prevalência; IC - Intervalo de Confiança;":Teste de Wald; TARV -terapia antiretroviral

(56,1\%) e o TG ( $45,5 \%)$, seguidos de PA ( $40,0 \%)$, CA (32,9\%) e GVJ (15,5\%). Já no critério da IDF, a CA alcançou 62,9\% de alteraçóes nas PVHIV avaliadas, seguindo os mesmo percentuais que o NCEP-ATPIII nos demais componentes. A GVJ representou a alteração metabólica menos prevalente em ambos os critérios.

No que se refere à distribuição dos pacientes de acordo com o número de componentes da $\mathrm{SM}$, constatou-se que de acordo com o critério da IDF $86,7 \%$ dos participantes têm pelo menos um componente que caracteriza esse distúrbio metabólico [nenhum componente (13,3\%), um (17,6\%), dois $(28,0 \%)$, três $(22,6 \%)$, quatro $(13,8 \%)$, cinco $(4,7 \%)]$. Já no critério do NCEP-ATPIII, 83,2\% tem pelo menos critério para a SM [nenhum componente $(16,8 \%)$, um $(23,3 \%)$, dois $(31,5 \%)$, três $(17,6 \%)$, quatro $(7,0 \%)$, cinco $(3,8 \%)]$.

A análise da concordância entre os critérios para avaliação da SM, IDF e NCEP-ATIII, obteve um coeficiente $A C=0,7493(\mathrm{p}<0,0001)$, considerando a concordância como substancial (Tabela 3 ).
Tabela 2. Componentes da síndrome metabólica em pessoas vivendo com HIV, de acordo com o sexo $(n=340)$

\begin{tabular}{|c|c|c|c|}
\hline Componentes da SM & $\begin{array}{l}\text { Média } \pm D P \\
\text { Masculino } \\
(n=197)\end{array}$ & $\begin{array}{c}\text { Média } \pm D P \\
\text { Feminino }(n= \\
143)\end{array}$ & $p$-value* \\
\hline Circunferência abdominal & $89,5 \pm 13,06$ & $88,9 \pm 18,71$ & 0,76 \\
\hline Glicemia venosa de jejum & $84,5 \pm 26,40$ & $96,7 \pm 57,96$ & 0,02 \\
\hline Triglicerídeos & $187,7 \pm 208,15$ & $154,6 \pm 113,36$ & 0,06 \\
\hline HDL-C & $42,0 \pm 13,76$ & $47,7 \pm 16,81$ & $<0,001$ \\
\hline Pressão Arterial Sistólica & $119,1 \pm 14,46$ & $115,0 \pm 15,96$ & 0,01 \\
\hline Pressão Arterial Diastólica & $78,3 \pm 9,47$ & $76,8 \pm 12,62$ & 0,25 \\
\hline
\end{tabular}

DP - desvio-padrão; *: T de Student; HDL-c: High Density Lipoproteins Cholesterol

Tabela 3. Concordância entre os critérios do National Cholesterol Education Program Adult Treatment Panel III (NCEP-ATPIII) e da International Diabetes Federation (IDF) para síndrome metabólica em pessoas vivendo com HIV $(n=340)$

\begin{tabular}{lccc}
\hline Critério & IDF $(-)$ & IDF $(+)$ & Total \\
\hline NCEP-ATPIII (-) & 201 & 42 & 243 \\
NCEP-ATPIII (+) & 05 & 92 & 97 \\
Total & 206 & 134 & 340 \\
Concordância & AC1 & EP & $p^{*}$ \\
NCEP-ATPIII vs IDF & 0,74 & 0,0356 & $<0,0001$ \\
\hline
\end{tabular}

(-): ausência de síndrome metabólica; (+): presença de síndrome metabólica; AC1: first-order agreement coefficient); EP: Erro Padrão; $p^{*}:<0,05$; vs: versus 


\section{Discussão}

Os resultados deste estudo evidenciam uma elevada prevalência de SM em PVHIV e associação ao sexo feminino e faixas etárias a partir dos 50 anos. As menores prevalências se associaram ao tempo de diagnóstico entre 2 a 10 anos. Houve concordância substancial entre os critérios IDF e NCEP-ATPIII.

$\mathrm{O}$ estudo possibilitou a identificação da prevalência e a concordância de critérios para a $S M$, contribuindo para a reflexão sobre a necessidade de avaliação dos componentes da síndrome durante o tratamento das PVHIV. Além de considerar a SM como uma ferramenta clínica no reconhecimento de indivíduos com maior vulnerabilidade à ocorrência de doenças cardiovasculares. Ademais, o estudo contribui para o desenvolvimento do conhecimento científico e para o debate sobre as necessidades de cuidado multiprofissional a essa população, bem como o papel do enfermeiro na avaliação cardiometabólica, da atenção primária até a terciária.

A SM tem recebido uma atenção cada vez maior, tanto pelo impacto de cada um dos seus componentes diagnósticos, quanto por agregar fatores de risco cardiovascular. $^{(16)}$ Nesse contexto, diversos fatores de risco para o desenvolvimento da SM têm sido identificados, porém há grande heterogeneidade nos resultados dos estudos. Esse fato está associado à variedade de critérios utilizados para avaliação da $\mathrm{SM}$ e as condiçôes sociodemográficas, comportamentais e clínicas das populaçóes investigadas.

A elevada prevalência encontrada no presente estudo entre as PVHIV em uso de TARV, corrobora com os achados de uma meta-análise que investigou 65 publicaçóes sobre SM em PVHIV nos continentes europeu, americano, asiático e africano. ${ }^{(9)}$ $\mathrm{O}$ estudo revelou que um terço da população mundial que vive com o HIV tem SM. ${ }^{(9)}$ Em pesquisas realizadas no continente africano houve prevalência entre 7,8-50,3\%, pelo critério IDF, e 7,2-61,6\% pelo critério NCEP-ATPIII. ${ }^{(17-19)}$ A concordância substancial entre os critérios da IDF e do NCEPATPIII dos nossos achados apoia o que tem sido evidenciado na literatura em estudos com PVHIV e outros grupos. ${ }^{(9,19-21)} \mathrm{O}$ resultado dessa concordância implica que esses critérios podem classificar o mes- mo indivíduo com SM, o que pode ser justificado pela utilização de quatro componentes metabólicos com o mesmo parâmetro de referência. A divergência dos critérios está na CA, que possui ponto de corte diferente, no critério da IDF é menor, comparado ao do NCEP-ATPIII. Outra diferença está na CA ser elemento obrigatório no critério da IDF para classificação da síndrome.

Os componentes da SM normalmente variam em suas taxas de ocorrência. Nesse estudo cerca de $80 \%$ das PVHIV apresentam pelo menos um componente alterado. As alteraçóes lipídicas e da CA estão entre os componentes metabólicos que mais prevalecem alterados, considerando os diferentes critérios utilizados. ${ }^{(9,22)}$ Em pesquisa internacional, esses componentes apresentam-se entre os primeiros fatores de risco para o desenvolvimento da SM. ${ }^{(17)} \mathrm{O}$ elevado número de componentes também é um fator preocupante por aumentar o risco de doenças cardiovasculares. ${ }^{(5)}$ Interessante destacar em nosso estudo que, apesar das diferenças entre homens e mulheres encontradas em alguns componentes metabólicos, as médias estão dentro dos parâmetros de normalidade das sociedades brasileiras responsáveis. ${ }^{(23,24)}$

Ademais, houve associação entre a SM e as mulheres vivendo com o HIV, o que pode ser atribuído à diferença do papel social que a mesma assume, com a dupla jornada de trabalho, que influência negativamente na qualidade de vida e repercute nos hábitos alimentares e na prática de atividade física. ${ }^{(9,17,25,26)}$ Além disso, fatores fisiológicos como a perda do efeito protetor dos hormônios femininos com o início da menopausa também são fatores importantes e que refletem a questão do gênero e a idade. ${ }^{(9,17,25,26)}$ Nessa perspectiva, a prevalência da SM aumenta com a idade. ${ }^{(9,17)}$ Outros estudos realizados na mesma região do Brasil apontam que as mulheres com HIV também possuem maior redistribuição anormal de gordura corporal (lipodistrofia) e maior frequência de casos de obesidade abdominal, quando comparados aos homens com HIV. ${ }^{(27,28)}$ Os mesmos autores, apesar de considerarem incertas as razóes da diferença sexual nas respostas metabólicas, reforçam a contribuição hormonal, seja pelo polimorfismo no gene do receptor de estrógeno ou pela resposta do corpo à liberação do hormônio do crescimento. ${ }^{(27,28)}$ 
Contudo, o resultado da prevalência da SM entre os que possuíam tempo de diagnóstico de 2 a 10 anos para o HIV pode ser justificada pelo fato dessa classificação estar entre a minoria dos entrevistados e, a maioria possuir mais de 10 anos de diagnóstico do $\operatorname{HIV}(45,5 \%)$.

Parte das associaçóes observadas entre a carga viral e o tempo de diagnóstico, podem ser explicadas por mecanismos de disfunção imunológica e inflamação crônica causados pela infecção do HIV, idade mais avançada e toxicidade relacionada à droga secundária ao uso prolongado, principalmente dos inibidores de protease. $^{(9,29,30)}$ Vale ressaltar que a própria infecção pelo HIV atua como um catalisador das alteraçóes lipídicas. $\mathrm{O}$ aumento da inflamação provocado pela presença do vírus, através da secreção de citocinas inflamatórias, danificam as células TCD4+. ${ }^{(31,32)}$

Diante do exposto, no contexto das PVHIV, as alterações metabólicas podem ser atribuídas não só a utilização de diferentes critérios diagnósticos, mas ao tempo de exposição à TARV, tempo de infecção pelo HIV, características étnicas, histórico familiar (contribuição genética), fatores ambientais, além dos hábitos alimentares e estilo de vida antes e após o tratamento. ${ }^{(9)}$

As informaçôes geradas evidenciam os critérios mais utilizados para a classificação da SM, a concordância entre eles, os fatores sociodemográficos e clínicos que podem se associar a síndrome, além dos componentes metabólicos que estão frequentemente alterados nas PVHIV, consequentemente, os dados obtidos neste estudo poderão subsidiar o estabelecimento de protocolos assistenciais com medidas voltadas a identificação, prevenção, tratamento e controle da SM nessa população. Assim, faz-se necessário o desenvolvimento de estratégias e programas educativos e terapêuticos com foco na promoção de hábitos saudáveis. Além da realização de estudos longitudinais que investiguem o comportamento das alteraçóes metabólicas nas PVHIV e estudos de intervenção que meçam o efeito das estratégias na melhoria dessas alteraçôes.

Destaca-se como limitação, que o desenho do estudo não permitiu que se conhecesse há quanto tempo os indivíduos apresentavam os critérios para $\mathrm{SM}$, se decorreu do tratamento medicamentoso ou foi anterior a este. Além disso, ressalta-se a escassez de informaçôes sobre a exposição cumulativa dos pacientes a cada medicamento ARV e a diversidade da sua história clínica e terapêutica.

$\mathrm{O}$ reconhecimento dos fatores de risco associados e a avaliação do perfil metabólico tornam-se necessários, uma vez que possibilitam a identificação dos que possuem alto risco cardiovascular, desempenhando um papel importante como marcador de distúrbios metabólicos, a fim de tratá-los com maior brevidade; além de evidenciar a importância de intervençóes na adoção de um estilo de vida saudável. Nesse contexto, o enfermeiro também tem papel fundamental junto à equipe de saúde para o desenvolvimento de estratégias educativas que favoreçam a promoção da saúde.

\section{Conclusão}

Assim, mediante os resultados apresentados verificou-se que a prevalência de SM em PVHIV foi elevada pelos dois critérios, mais especialmente pelo IDF. As maiores prevalências de SM foram associadas ao sexo feminino, e faixas etárias a partir dos 50 anos, enquanto que, no tempo de diagnóstico entre 2 a 10 anos, as prevalências foram menores. Houve concordância substancial entre os critérios IDF e NCEP-ATPIII, o implica que esses critérios podem classificar o mesmo indivíduo com SM e assim, há possibilidade de intercambio entre eles.

\section{Agradecimentos}

Agradecemos ao Ministério da Ciência, Tecnologia e Inovação - Conselho Nacional de Desenvolvimento Científico e Tecnológico - CNPq (no 455912/20149) - Chamada Universal - MCTI/CNPq $n^{\circ}$ 14/2014, pelo apoio na realização deste estudo.

\section{Colaborações}

Costa CRB, Melo ES, Oliveira LB, Moreira RSL, Gir E e Reis RK colaboraram com a concepção do estudo, análise e interpretação dos dados, redação 
do artigo, revisão intelectual do conteúdo e aprovação da versão final a ser publicada.

\section{Referências}

1. Grinsztejn B, Luz PM, Pacheco AG, Santos DV, Velasque L, Moreira RI, et al. Changing mortality profile among HIV-infected patients in Rio de Janeiro, Brazil: shifting from AIDS to non-AIDS related conditions in the HAART era. PLoS One. 2013;8(4):e59768.

2. Astolfo S, Kehrig RT, Oliveira LR. Availability of resources in Brazilian National Health System outpatient services for people living with HIV in Mato Grosso, Brazil, 2016. Epidemiol Serv Saude. 2018;27(3):e2017406.

3. Join United Nations Programme on HIV/Aids (UNAIDS). UNAIDS DATA 2019. Geneva: UNAIDS; 2019.

4. Brasil. Ministério da Saúde. Relatório de Monitoramento Clínico do HIV. Brasília (DF): Ministério da Saúde; 2019.

5. Deeks SG, Lewin SR, Havlir DV. The end of AIDS: HIV infection as a chronic disease. Lancet. 2013;382(9903):1525-33.

6. Guira O, Tiéno H, Diendéré AE, Sagna Y, Diallo I, Yaméogo B, et al. Features of Metabolic Syndrome and Its Associated Factors during Highly Active Antiretroviral Therapy in Ouagadougou (Burkina Faso). J Int Assoc Provid AIDS Care. 2016;15(2):159-63.

7. Sobieszczyk ME, Werner L, Mlisana K, Naicker N, Feinstein A, Gray CM, et al. Síndrome metabólica após a aquisição do hiv em mulheres SulAfricanas. J Acquir Immune Defic Syndr. 2016;73(4):438-45.

8. Santilli F, D’Ardes D, Guagnano MT, Davi G. Metabolic syndrome: sexrelated cardiovascular risk and therapeutic approach. Curr Med Chem. 2017;24(24):2602-27.

9. Nguyen KA, Peer N, Mills EJ, Kengne AP. A meta-analysis of the metabolic syndrome prevalence in the global hiv-infected population. PLoS One. 2016;11(3):e0150970.

10. Malachias MV, Gomes MA, Nobre F, Alessi A, Feitosa AD, Coelho EB. 7th brazilian guideline of arterial hypertension: chapter 2 - diagnosis and classification. Arq Bras Cardiol. 2016;107(3 Suppl 3):7-13.

11. World Health Organization (WHO). Waist circumference and waist-hip ratio: report of a WHO expert consultation. Geneva: WHO; 2008.

12. Grundy SM, Cleeman JI, Daniels SR, Donato KA, Eckel RH, Franklin BA, et al.; American Heart Association; National Heart, Lung, and Blood Institute. Diagnosis and management of the metabolic syndrome: an American Heart Association/National Heart, Lung, and Blood Institute Scientific Statement. Circulation. 2005;112(17):2735-52.

13. International Diabetes Federation (IDF). The IDF consensus worldwide definition of the metabolic syndrome. Belgium: IDF; 2006.

14. Associação Brasileira para o Estudo da Obesidade e da Síndrome Metabólica (ABESO). Diretrizes Brasileiras de Obesidade 2016. 4a ed. São Paulo: ABESO; 2016.

15. Landis JR, Koch GG. The measurement of observer agreement for categorical data. Biometrics. 1977;33(1):159-74.

16. Drelichowska J, Kwiatkowska W, Knysz B, Witkiewicz W. Metabolic syndrome in HIV-positive patients. HIV AIDS Rev. 2015;14(2):35-41.
17. Hirigo AT, Tesfaye DY. Influences of gender in metabolic syndrome and its components among people living with HIV virus using antiretroviral treatment in Hawassa, southern Ethiopia. BMC Res Notes. 2016;9(1):145.

18. Obirikorang C, Quaye L, Osei-Yeboah J, Odame EA, Asare I. Prevalence of metabolic syndrome among HIV-infected patients in Ghana: A crosssectional study. Niger Med J. 2016;57(2):86-90.

19. Nguyen KA, Peer N, de Villiers A, Mukasa B, Matsha TE, Mills EJ, et al. Metabolic syndrome in people living with human immunodeficiency virus: an assessment of the prevalence and the agreement between diagnostic criteria. Int J Endocrinol. 2017;2017:1613657.

20. Saad MA, Cardoso GP, Martins WA, Velarde LG, Cruz Filho RA. Prevalence of metabolic syndrome in elderly and agreement among four diagnostic criteria. Arq Bras Cardiol. 2014;102(3):263-9.

21. Adnan M, Rahat T, Hashmat N, Ali Z. Metabolic syndrome; agreement between diagnostic criteria among type 2 diabetes mellitus patients. Prof Med J. 2017;24(4):539-44.

22. Mbugua SM, Kimani ST, Munyoki G. Metabolic syndrome and its components among university students in Kenya. BMC Public Health. 2017;17(1):909.

23. Faludi AA, Izar MC, Kerr SJ, Marte CA, Bianco HT, Afiune NA, et al. Atualização da Diretriz Brasileira de Dislipidemias e Prevenção da Aterosclerose - 2017. Arq Bras Cardiol. 2017;109(2 Suppl1):1-76.

24. Diretrizes da Sociedade Brasileira de Diabetes 2017-2018. São Paulo: Editora Clannad; 2017. [organizado por José Egídio Paulo de Oliveira, Renan Magalhães Montenegro Junior, Sérgio Vencio].

25. Galvão MT, Soares LL, Pedrosa SC, Fiuza ML, Lemos LA. Quality of life and adherence to antiretroviral medication in people with HIV. Acta Paul Enferm. 2015;28(1):48-53.

26. AkI LD, Valadares AL, Moraes MJ, Pinto-Neto AM, Lagrutta B, CostaPaiva L. Metabolic syndrome in HIV-infected middle-aged women on antiretroviral therapy: prevalence and associated factors. Braz J Infect Dis. 2017;21(3):263-9.

27. Alves TC, Moraes C, Santos AP, Venturini ACR, Santana RC, Navarro $A M$, et al. Increased chance of metabolic syndrome in women living with HIV/AIDS and lipodystrophic syndrome. Medicina (Ribeirão Preto. 2016; 49(5):421-8.

28. Beraldo RA, Santos AP, Guimarães MP, Vassimon HS, Paula FJ, Machado DR, et al. Body fat redistribution and changes in lipid and glucose metabolism in people living with HIV/AIDS. Rev Bras Epidemiol. 2017;20(3):526-36.

29. Ximenes RA, Lacerda HR, Miranda-Filho DB, Albuquerque MF, Montarroyos UR, Turchi MD, et al. Comparison between potential risk factors for cardiovascular disease in people living with HIV/AIDS in areas of Brazil. J Infect Dev Ctries. 2015;9(9):988-96.

30. Brasil. Ministério da Saúde. Secretaria de Vigilância em Saúde, Departamento de Vigilância, Prevenção e Controle das Infecções Sexualmente Transmissíveis, do HIV/Aids e das Hepatites Virais. Protocolo Clínico e Diretrizes Terapêuticas para Manejo da Infecção pelo HIV em adultos. Brasília (DF): Ministério da Saúde; 2018.

31. Nix LM, Tien PC. Metabolic syndrome, diabetes, and cardiovascular risk in HIV. Curr HIV/AIDS Rep. 2014;11(3):271-8.

32. Dada A0, Oshodi TT, Ajie 10, Onyenekwu CP. Prevalence of insulin resistance among patients attending the HIV clinic in a Nigerian tertiary hospital. Diabetes Metab Syndr. 2017;11 Suppl 2:S607-10. 